\title{
Gene expression in human brown adipose tissue
}

\author{
PER-ARNE SVENSSON ${ }^{1}$, MARGARETA JERNÅ ${ }^{1}$, KAJSA SJÖHOLM ${ }^{1}$, JENNY M. HOFFMANN $^{1}$, \\ BENGT E. NILSSON ${ }^{2}$, MAGNUS HANSSON $^{3}$ and LENA M.S. CARLSSON ${ }^{1}$ \\ ${ }^{1}$ Sahlgrenska Center for Cardiovascular and Metabolic Research, Department of Molecular and Clinical Medicine, \\ Departments of ${ }^{2}$ Surgery and ${ }^{3}$ Clinical Pathology and Cytology, The Sahlgrenska Academy, \\ University of Gothenburg, SE-41345 Gothenburg, Sweden
}

Received August 21,2010; Accepted October 15, 2010

DOI: $10.3892 /$ ijmm.2010.566

\begin{abstract}
Brown adipose tissue (BAT) has profound effects on body weight and metabolism in rodents. Recent reports show that human adults have significant amounts of BAT. Our aim was to study the gene expression profile of human BAT. Biopsies of adipose tissue with brown-red color and subcutaneous white adipose tissue (WAT) were obtained from 24 patients undergoing surgery in the thyroid region. Intrascapular BAT and epididymal WAT biopsies were obtained from 10 mice. Expression was analyzed by DNA microarray, real-time PCR and immunohistochemistry. Using the expression of the brown adipocyte-specific gene uncoupling protein 1 (UCP1) as a marker, approximately half of the human brown-red adipose tissue biopsies taken in the thyroid region contained BAT, and the presence of cells with brown adipocyte morphology was also verified by histology. Microarray analysis of 9 paired human BAT and WAT samples showed that 17 genes had at least a 4-fold higher expression in BAT compared to WAT and five of them (CKMT1, KCNK3, COBL, HMGCS2, TGM2) were verified using real-time $\mathrm{PCR}(\mathrm{P}<0.05$ for all). In addition, immunohistochemistry showed that the UCP1, KCNK3 and CKMT1 proteins are expressed in brown adipocytes. Except for UCP1 and $\mathrm{KCNK} 3$, the genes overexpressed in human BAT were not overexpressed in mouse BAT compared to mouse WAT. Our analysis identified genes that are differentially expressed in human BAT compared to WAT. The results also show that there are species-specific differences in BAT gene expression and this emphasizes the need for further molecular characterization of human BAT to clarify the mechanisms involved in regulated heat production in humans.
\end{abstract}

Correspondence to: Per-Arne Svensson, Sahlgrenska Center for Cardiovascular and Metabolic Research, Department of Molecular and Clinical Medicine, University of Gothenburg, Vita stråket 15, SE-41345 Gothenburg, Sweden

E-mail: per-arne.svensson@gu.se

Key words: brown adipose tissue, white adipose tissue, gene expression profiling, uncoupling protein 1 , human, mouse

\section{Introduction}

There are two types of adipose tissue, white (WAT) and brown (BAT) adipose tissue, that have different biological roles. WAT is the main site for energy storage and it is also an important endocrine organ that signals to other tissues via the release of adipokines (1). The main role of BAT appears to be the maintenance of body temperature by converting chemical energy to heat through the uncoupling of oxidative phosphorylation (2).

In humans, WAT is an easily accessible tissue that has been subjected to intensive research because of its proposed role in obesity and metabolic dysfunction. Human BAT, on the other hand, has been regarded as a tissue that is only present in small children and at anatomical sites that are difficult to reach. A few older studies have shown that BAT is present in some adult humans $(3,4)$. However, these findings have largely been ignored and it has been generally accepted that BAT disappears early in life and therefore lacks importance in human adults. A number of recent reports have challenged this idea and it is now evident that many adults have significant amounts of BAT (5). Using uptake of labeled glucose in combination with positron emission tomography (PET), BAT has been detected in adult humans, and cold exposure has revealed that this tissue can be activated $(5,6)$. BAT activity appears to be higher in lean than in obese subjects $(7,8)$ and correlates with resting metabolic rate (8), suggesting that BAT may be a hitherto unrecognized regulator of adult human metabolism and energy expenditure.

At the molecular level, extensive studies in rodents have highlighted the key role of uncoupling protein 1 (UCP1) in brown adipocytes. UCP1 uncouples the ATP synthase and provides an alternative pathway by which protons can reenter the mitochondrial matrix to generate heat (9). In rodents, UCP1 is exclusively expressed by brown adipocytes and UCP1 expression is widely used as a marker of BAT (10). Recent reports show that UCP1 is also highly expressed in human BAT and immunohistochemistry has confirmed that UCP1 protein expression is localized to cells with brown adipocyte morphology $(11,12)$.

In addition to UCP1, studies in rodents have pinpointed several other genes of importance for the development and function of BAT, such as FOXC2 (13), PGC-alpha (14), PRDM16 (15,16), DIO2 (17), ADRB3 (18) and BMP7 (19). 
The expression of some of these genes has been confirmed in human BAT (11), suggesting that there are similarities in BAT function between humans and rodents. However, the molecular characterization of human BAT has only begun. The present study was, therefore, undertaken to explore gene expression in human BAT.

\section{Materials and methods}

The regional ethics committee in Gothenburg approved these studies. All study subjects received written and oral information before providing written informed consent.

Subjects and samples. Twenty-four patients scheduled for surgery in the thyroid region were included in this study (Table I). Biopsies of adipose tissue of brown-red color were obtained from an area close to the isthmus region of the thyroid gland. Some biopsies were cut in half to allow for both histological/immunohistochemical evaluation and expression analysis. In each patient, biopsies were also taken from the subcutaneous depot in the surgical incision area and were referred to as WAT.

Histology and immunohistochemistry. Biopsies were fixed overnight at $4{ }^{\circ} \mathrm{C}$ in a solution containing $4 \%$ formaldehyde in $0.1 \mathrm{M}$ phosphate buffer, $\mathrm{pH} 7.0$, embedded in paraffin, sectioned and stained with hematoxylin and eosin. For immunohistochemistry, sections were deparaffinized and rehydrated. Antigen retrieval was performed using the 2100 Retriever (Biocare Medical, Concord, CA, USA). Staining was performed using the Elite ABC kit (Vector Laboratories, Burlingame, CA, USA) and antibodies against UCP1 (1:1500, Sigma Aldrich, St. Louis, MO, USA), KCNK3 (1:25, Sigma Aldrich), or CKMT1A (1:1300, ProteinTech Group, Chicago, IL, USA).

Mouse adipose tissue. Paired biopsies of epididymal WAT and intrascapular BAT were obtained from ten female C57BL/6 mice (13 week-old) fed regular chow diet.

RNA preparation and DNA microarray analysis. Samples for RNA preparation were snap frozen in liquid nitrogen, and stored at $-80^{\circ} \mathrm{C}$ until analyzed. Total RNA was prepared using the phenol-chloroform extraction method of Chomczynski and Sacchi (20). After further purification with RNeasy clean-up columns (Qiagen), the RNA concentration was measured spectrophotometrically. Gene expression was evaluated using the Human Genome U133Plus2.0 DNA microarray (Affymetrix). Preparation of cRNA and hybridization were performed according to standard Affymetrix protocols as previously described (21).

Microarray data mining. Genes specifically expressed in human BAT compared to WAT were identified as those with a BAT/WAT signal ratio $>4$ and a $\mathrm{P}<0.01$ in a paired student t-test (two-sided). When multiple probes for the same gene were present, the probe with the highest expression in BAT was used for the analysis.

Real-time PCR analysis of gene expression. Reagents for real-time PCR analysis of human UCP1 (Hs00222453_m1),
Table I. Patient characteristics.

\begin{tabular}{|c|c|c|c|c|}
\hline Patient & Sex & $\begin{array}{c}\text { Age } \\
\text { (years) }\end{array}$ & $\begin{array}{c}\text { BMI } \\
\left(\mathrm{kg} / \mathrm{m}^{2}\right)\end{array}$ & Reason for surgery \\
\hline 1 & Male & 21 & 23.6 & Hyperparathyroidism \\
\hline 2 & Female & 39 & 23.3 & Non-toxic goiter \\
\hline 3 & Female & 75 & 23.5 & Thyrotoxicosis \\
\hline 4 & Male & 23 & 20.2 & Thyrotoxicosis \\
\hline 5 & Female & 66 & 34.8 & Non-toxic goiter \\
\hline 6 & Male & 76 & 25.3 & Thyrotoxicosis \\
\hline 7 & Female & 28 & 26.4 & Non-toxic goiter \\
\hline 8 & Male & 68 & 26.0 & Thyrotoxicosis \\
\hline 9 & Female & 60 & 20.3 & Benign thyroid cyst \\
\hline 10 & Female & 71 & 19.0 & Hyperparathyroidism \\
\hline 11 & Female & 51 & 27.8 & Non-toxic goiter \\
\hline 12 & Female & 79 & 26.4 & Non-toxic goiter \\
\hline 13 & Female & 44 & 21.9 & Thyrotoxicosis \\
\hline 14 & Female & 37 & 21.4 & Non-toxic goiter \\
\hline 15 & Female & 59 & 21.6 & Hyperparathyroidism \\
\hline 16 & Female & 71 & 26.4 & Hyperparathyroidism \\
\hline 17 & Female & 65 & 21.2 & Hyperparathyroidism \\
\hline 18 & Female & 50 & 23.1 & Non-toxic goiter \\
\hline 19 & Female & 50 & 25.9 & Non-toxic goiter \\
\hline 20 & Male & 43 & 35.5 & Non-toxic goiter \\
\hline 21 & Female & 70 & 31.3 & Non-toxic goiter \\
\hline 22 & Female & 26 & 25.6 & Thyroid cancer \\
\hline 23 & Female & 76 & 25.7 & Non-toxic goiter \\
\hline 24 & Female & 32 & 23.8 & Thyrotoxicosis \\
\hline
\end{tabular}

KCNK3 (Hs00605529_m1), CKMT1A/1B (Hs00179727_m1), COBL (Hs00391205_m1), HMGCS2 (Hs00194145_m1), TGM2 (Hs00190278_m1) and the endogenous reference genes RPLP0 (Hs99999902_m1) (Assays-on-Demand, TaqMan Reverse Transcriptase reagents, and TaqMan Universal PCR Master Mix) were from Applied Biosystems (Foster City, CA) and used according to the manufacturer's protocol. cDNA corresponding to $5 \mathrm{ng}$ of RNA per reaction was used for realtime PCR analysis. Specific products were amplified and detected with the ABI Prism 7900HT sequence detection system (Applied Biosystems) using default cycle parameters. A standard curve obtained by serial dilution of pooled adipose tissue cDNA was plotted for each primer-probe set. All standards and samples were analyzed in triplicate. Mouse RNA was analyzed as described above using assays for Ckmt1 (Mm00438216_m1), Kcnk3 (Mm00807036_m1), Cobl (Mm00552805_m1), Tgm2 (Mm00436980_m1) and Hmgcs2 (Mm00550050_m1). The TATA box-binding protein (Tbp, Mm00446971_m1) was used as the endogenous reference gene (16).

Statistical analysis. Differences in gene expression between BAT and WAT were analyzed by paired Student's t-test (two-sided). P-values of $<0.05$ were considered statistically significant. 

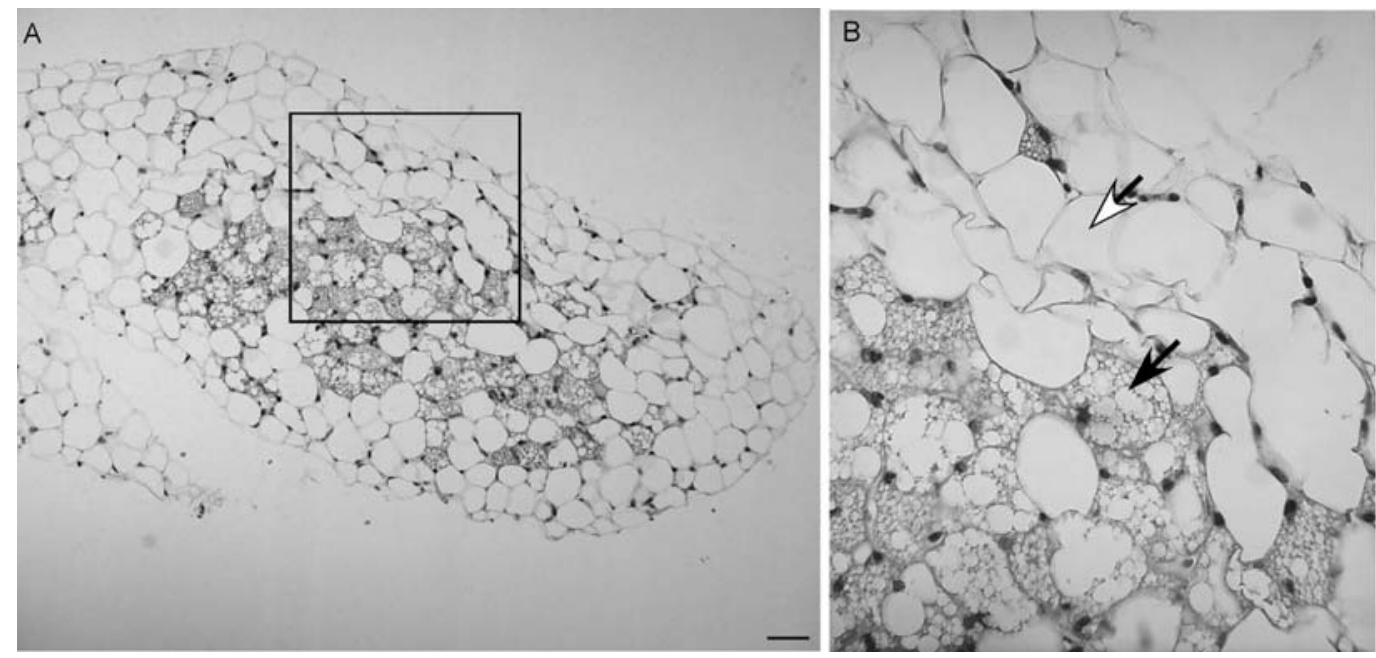

Figure 1. (A) Microscopic picture of hematoxylin and eosin stained human UCP1-positive perithyroid adipose tissue at x100 magnification. The bar represents $100 \mu \mathrm{m}$. (B) x400 magnification of the same section. The section contains a mixture of white adipocytes with a single large lipid droplet (white arrow) and brown adipocytes with multiple lipid droplets (black arrow).

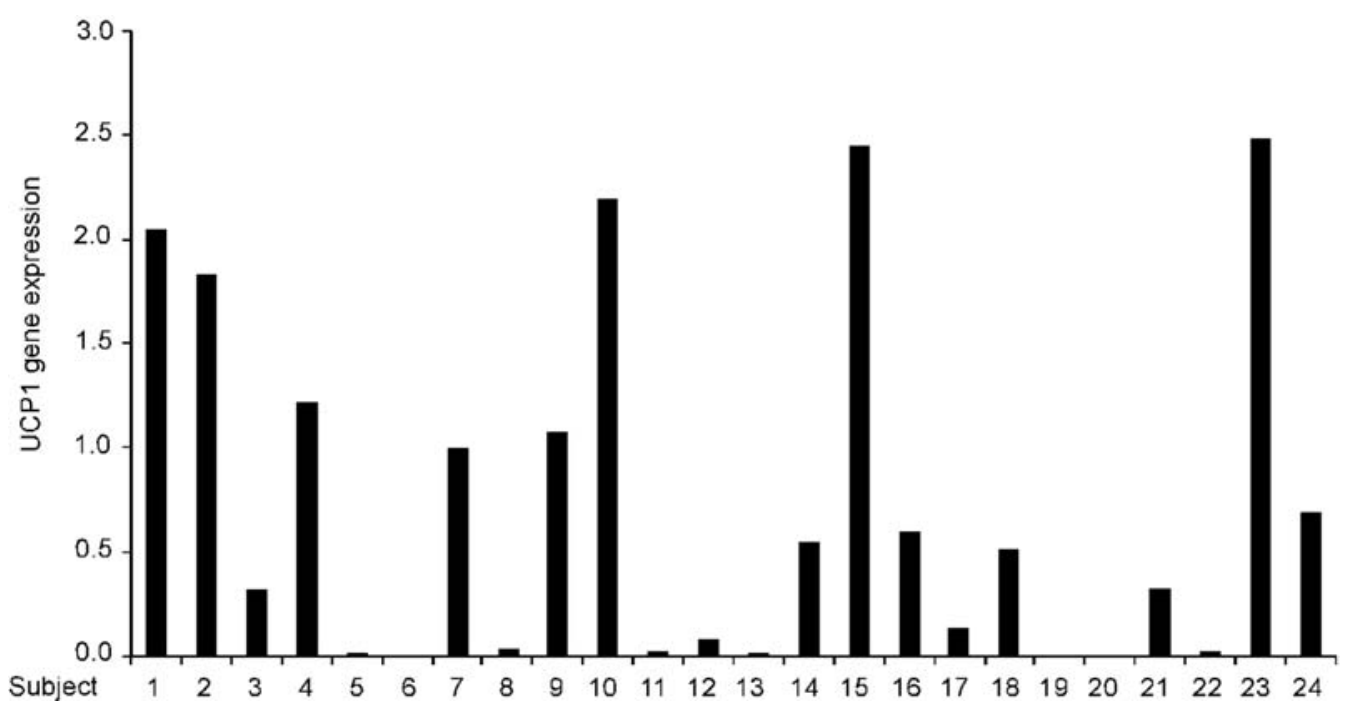

Figure 2. UCP1 gene expression in brown-red samples of perithyroid adipose tissue from 24 patients. UCP1 expression was analyzed by real-time PCR and related to the reference gene RPLP0. Data shown are individual expression levels.

\section{Results}

Characterization of human perithyroid adipose tissue biopsies. Paired adipose tissue biopsies with brown-red appearance from the perithyroid region and s.c. WAT were obtained from 24 patients undergoing surgery in the thyroid region. Histological examination showed the presence of cells with brown adipocyte morphology dispersed between white adipocytes (Fig. 1) in biopsies with brown-red appearance. To verify the presence of brown adipocytes, the expression of the BAT-specific gene UCP1 was analyzed in all biopsies. UCP1 expression was undetectable in all WAT samples (data not shown). In approximately half of the brown-red biopsies, UCP1 expression was clearly detectable (Fig. 2), and for the purpose of this study, biopsies were classified as BAT if UCP1 expression was $>0.5$ relative to the reference gene RPLP0.
Gene expression in BAT. To identify genes that are differentially expressed in human BAT and WAT, samples from patient nos. 1, 2, 4, 9, 15, 16, 18, 23 and 24 were analyzed by microarray (Table I). This analysis confirmed that the UCP1 gene was overexpressed (>60-fold, Table II) in samples classified as BAT. Further analysis of the expression profiles, using the criteria described in Materials and methods, showed that 16 other genes were also expressed at higher levels in BAT compared to WAT samples (Table II). According to Gene Ontology (GO) analysis (www.ncbi.nlm.nih.gov/gene; accessed 2010-04-07), five of these genes encode proteins that have their main function in mitochondria, three encode proteins localized to the plasma membrane and three encode secreted proteins (Table II).

To verify the microarray data, five of the genes (KCNK3, CKMT1A/B, COBL, TGM2, HMGCS2) were analyzed by 
Table II. Genes overexpressed in human BAT compared to WAT.

\begin{tabular}{llclc}
\hline Gene title & Gene symbol & Signal ratio & P-value & GO cellular component \\
\hline Uncoupling protein 1 & UCP1 & 61.1 & $4.9 \times 10^{-7}$ & Mitochondrion \\
Creatine kinase, mitochondrial 1A/B & CKMT1A/B & 16.2 & $7.1 \times 10^{-6}$ & Mitochondrion \\
Potassium channel, subfamily K, member 3 & KCNK3 & 11.3 & 0.00063 & Plasma membrane \\
Immunoglobulin heavy constant mu & IGHM & 10.0 & 0.0084 & Extracellular region \\
Cordon-bleu & COBL & 8.0 & $7.8 \times 10^{-6}$ & NA \\
Cytoplasmic FMR1 interacting protein 2 & CYFIP2 & 7.4 & 0.00054 & Cytoplasm \\
Cytochrome P450, family 1, subfamily A, & CYP1A2 & 5.8 & 0.00025 & Endoplasmic reticulum \\
polypeptide 2 & & & & Myosin filament \\
Myosin, heavy chain 11, smooth muscle & MYH11 & 5.6 & 0.0042 & Extracellular region \\
Angiotensinogen & AGT & 5.5 & 0.00021 & Mitochondrion \\
Creatine kinase, mitochondrial 2 & CKMT2 & 5.2 & 0.00049 & Mitochondrion \\
Transglutaminase 2 & TGM2 & 5.1 & 0.0017 & Mitochondrion \\
Arginase, type II & ARG2 & 4.9 & 0.00015 & Plasma membrane \\
Carbonic anhydrase XII & CA12 & 4.9 & 0.00013 & Mitochondrion \\
3-Hydroxy-3-methylglutaryl-coenzyme A & HMGCS2 & 4.7 & 0.00019 & Extracellular region \\
synthase 2 (mitochondrial) & & & & Plasma membrane \\
Complement component 4A/B & C4A/B & 4.5 & 0.00042 & NA \\
Integrin, alpha 8 & ITGA8 & 4.5 & 0.0092 & 0.0087 \\
Immunoglobulin lambda variable 2-14 & IGLV2-14 & 4.4 & &
\end{tabular}

${ }^{a}$ Calculated as mean BAT microarray signal/mean WAT microarray signal. NA, not available
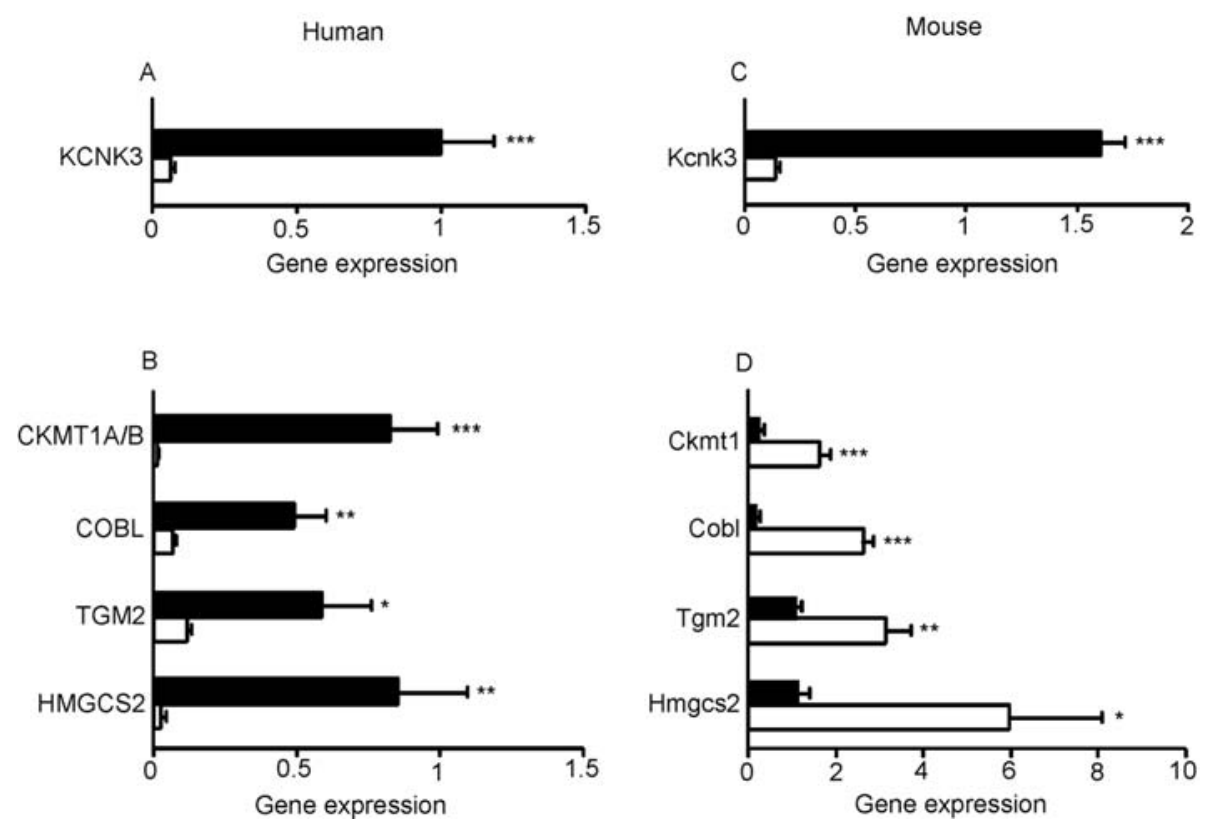

Figure 3. Real-time PCR verification of genes identified as highly expressed in human BAT compared to WAT in the microarray analysis and the expression of the mouse homologues in mouse BAT and WAT. Expression of KCNK3 (A) and CKMT1A/B, COBL, TGM2, HMGCS2 (B) in human BAT and WAT $(n=11)$. Expression of the corresponding mouse genes $(C$ and $D ; n=10)$. The expression of each gene was related to the reference genes RPLP0 (human) and Tbp (mouse). Data are presented as mean \pm SEM. ${ }^{*} \mathrm{P}<0.05,{ }^{* *} \mathrm{P}<0.01$ and ${ }^{* * *} \mathrm{P}<0.001$.

real-time PCR in paired BAT and WAT samples from patient nos. 1, 2, 4, 7, 9, 10, 14, 15, 16, 18, and 23 (Table I). The results confirmed that the expression of these genes was at least 4-fold higher in BAT compared to WAT (Fig. 3A and B).
Furthermore, the expression of the five genes correlated with the expression of UCP1 $(\mathrm{KCNK} 3$, rho $=0.918, \mathrm{P}=0.00007$; CKMT1A/B, rho=0.682, $\mathrm{P}=0.02 ; \mathrm{COBL}, \mathrm{rho}=0.900, \mathrm{P}=0.0002$; TGM2, rho=0.755, $\mathrm{P}=0.007$; HMGS2, rho=0.655, $\mathrm{P}=0.03$ ). 

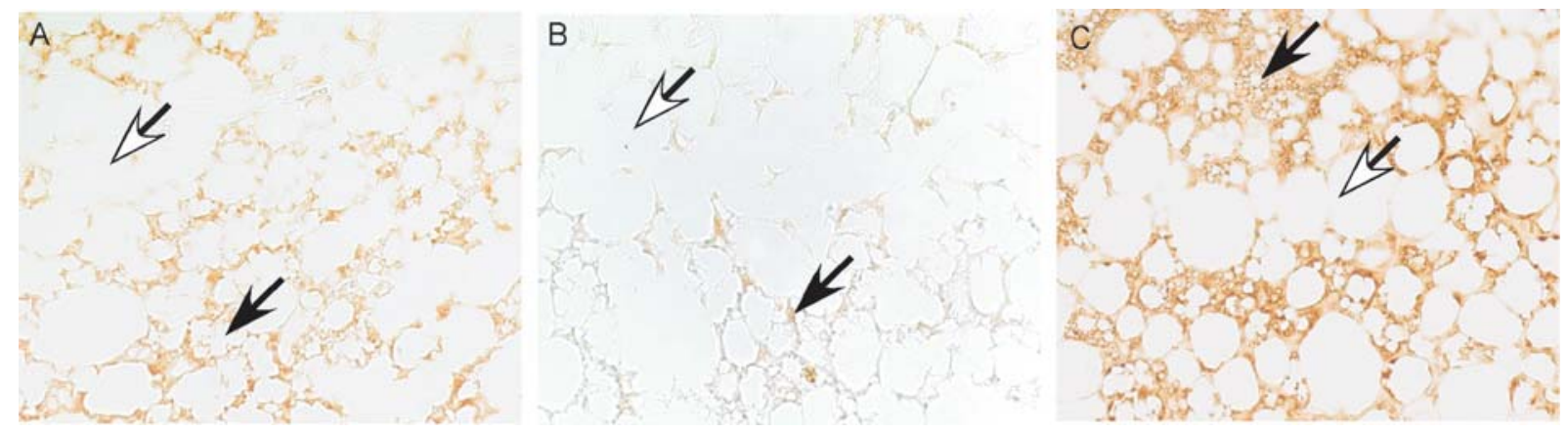

Figure 4. Immunohistochemical analysis of UCP1 (A), KCNK3 (B) and CKMT1A/B (C) in human perithyroid adipose tissue. White adipocytes with a single large lipid droplet (white arrow) and brown adipocytes with multiple lipid droplets (black arrow) are indicated in each figure.

Analysis of the mouse homologues of these genes in mouse BAT and WAT samples showed that Kcnk3 is overexpressed also in mouse BAT (Fig. 3C). However, in contrast to the expression pattern found in human samples, Cobl, Ckmt1, Tgm2 and Hmgcs2 expression was significantly higher in mouse WAT compared to BAT (Fig. 3D).

Immunohistochemical analysis of UCP1, KCNK3 and $C K M T 1 A / B$. To confirm that the overexpression of KCNK3 and CKMT1A/B in human BAT was due to their expression in brown adipocytes we performed immunohistochemical analysis. Using antibodies against UCP1, KCNK3 and CKMT1A/B, intensive staining was observed in cells with brown adipocyte morphology compared to white adipocytes on the same sections (Fig. 4) and in the negative control sections where the primary antibody was omitted (data not shown).

\section{Discussion}

Research efforts to understand the cause of obesity and related metabolic diseases have been focused on the cross-talk between WAT, liver, skeletal muscle, pancreas and the central nervous system. Recent reports describing the surprisingly large amount of BAT in human adults introduces a new player on this scene $(5,7,8,11)$. The belief that BAT disappears soon after birth in humans has led to the assumption that mitochondrial uncoupling and regulated heat production are unimportant in human adults. Instead, recent results indicate that human BAT may contribute to whole body metabolism in adults (8). However, the magnitude of its impact and the mechanisms involved are far from clear.

Brown adipocytes are specialized cells that express UCP1 and therefore, have the ability to uncouple the mitochondrial oxidative phosphorylation from ATP production and to dissipate energy as heat (10). Using UCP1 as a marker for brown adipocytes, we found that adipose tissue biopsies from the perithyroid region contained significant amounts of BAT. This is in line with the study by Zingaretti et al in which adipose tissue biopsies obtained during surgery in the thyroid region displayed distinct islands of UCP1 immunoreactive brown adipocytes (12). In our samples, there was a mixture of brown and white adipocytes, and this has also been observed in other studies on human BAT $(8,11,12)$ and in a human brown adipocyte tumor (hibernoma) (22). Thus, it is difficult to obtain samples of human BAT that are free from white adipocytes, and this complicates the characterization of human BAT gene expression. Microarray analysis detects signals derived from all cell types in the samples. To identify BAT specific genes, we therefore compared microarray expression profiles from UCP1-positive BAT samples with UCP1-negative WAT samples from the same patient. This approach identified several genes that were highly expressed in human BAT and expressed at much lower levels in human WAT, suggesting that they are involved in processes that occur specifically in the human brown adipocyte. This idea was supported by our GO analysis, which showed that several of the genes have their main function in mitochondria. In addition, the immunohistochemistry showed that genes that were highly expressed in our human BAT samples (UCP1, KCNK3 and CKMT1A/B) were indeed produced in brown adipocytes.

So far, essentially all knowledge on BAT function and development is derived from small mammals where it is well established that BAT is a key metabolic tissue throughout life that helps to maintain body temperature and which regulates energy expenditure and affects body weight (10). Such studies have clarified the uncoupling process and have started to unravel the cellular precursors and differentiation process of brown adipocytes (10). In contrast, very little is known about human BAT at the molecular level. However, using biopsies from three human subjects, Virtanen et al measured the expression of DIO2, PGC-alpha, PRDM16 and ADRB3, genes implicated in BAT function in mice, and found higher expression in human BAT compared to WAT (11), suggesting similarities between humans and rodents. However, in our study DIO2, PGC-alpha, PRDM16 and ADRB3 did not fulfill the stringent selection criteria for BAT specific genes although they tended to be more highly expressed in human BAT compared to human WAT (data not shown). Furthermore, several of the human BAT genes that we identified had higher expression in WAT compared to BAT in the mouse, emphasizing that there are species-specific differences in BAT gene expression.

Despite intensive research, there is still no efficient medical treatment for obesity and bariatric surgery is currently the only option to obtain long-term weight loss in obese patients (23). Non-surgical treatments are urgently needed and stimulation of adaptive thermogenesis is an attractive strategy to increase energy expenditure. The idea to increase the 
uncoupling process to burn off excess calories as heat is not new. The mitochondrial uncoupling agent 2,4-dinitrophenol was used extensively in the 1930s to treat obesity because of its ability to increase metabolic rate. Although this treatment was discontinued because of side-effects (24), it reduced body weight and therefore, provides proof-of-concept for controlled targeting of mitochondrial uncoupling. Increased heat production at the expense of energy storage in WAT may be achieved either by stimulating existing BAT or by the induction of more brown adipocytes. BAT is activated by cold, a function mediated by the sympathetic nervous system via the B-3 adrenergic receptor in rodents (10). The pharmaceutical industry has developed agonists of the $\beta-3$ adrenergic receptor based on the assumption that such compounds would also increase energy expenditure in man. Although agonists of this receptor effectively reduce obesity in rodents, they have failed in clinical trials (25). Such failures, together with our observations that there are species-specific differences in BAT gene expression, suggest that research on human samples is needed to clarify the molecular mechanisms that could be used for drug development (26).

In conclusion, we have identified genes that are differentially expressed in human BAT and WAT and we show that there are species-specific differences in BAT gene expression. Our results highlight the importance of the molecular characterization of human BAT in order to understand its function and to identify mechanisms that can be used for the development of anti-obesity drugs that increase energy expenditure.

\section{Acknowledgements}

The authors would like to acknowledge the technical assistance of Susanne Hering, Maja Olsson, Vilborg Palsdottir, Camilla Glad, Magdalena Taube and Carina Karlsson. This study was supported by grants from the Swedish Research Council (K2010-55X-11285-13, K2008-65X-20753-01-4, K200571X-15424-01A); the Swedish Foundation for Strategic Research, Sahlgrenska Center for Cardiovascular and Metabolic Research; the Swedish Diabetes Foundation; AstraZeneca; the Jeansson Foundations; the Ake Wiberg Foundation; the VINNOVA-VINNMER program; the Dr P. Håkanssons Foundation and the Swedish federal government under the LUA/ALF agreement.

\section{References}

1. Trayhurn P and Wood IS: Signalling role of adipose tissue: adipokines and inflammation in obesity. Biochem Soc Trans 33: 1078-1081, 2005.

2. Costford S, Gowing A and Harper ME: Mitochondrial uncoupling as a target in the treatment of obesity. Curr Opin Clin Nutr Metab Care 10: 671-678, 2007.

3. Heaton JM: The distribution of brown adipose tissue in the human. J Anat 112: 35-39, 1972.

4. Huttunen P, Hirvonen J and Kinnula V: The occurrence of brown adipose tissue in outdoor workers. Eur J Appl Physiol Occup Physiol 46: 339-345, 1981.

5. Nedergaard J, Bengtsson T and Cannon B: Unexpected evidence for active brown adipose tissue in adult humans. Am J Physiol Endocrinol Metab 293: E444-E452, 2007.
6. Hany TF, Gharehpapagh E, Kamel EM, Buck A, Himms-Hagen J and von Schulthess GK: Brown adipose tissue: a factor to consider in symmetrical tracer uptake in the neck and upper chest region. Eur J Nucl Med Mol Imaging 29: 1393-1398, 2002.

7. Cypess AM, Lehman S, Williams G, et al: Identification and importance of brown adipose tissue in adult humans. N Engl J Med 360: 1509-1517, 2009.

8. van Marken Lichtenbelt WD, Vanhommerig JW, Smulders NM, et al: Cold-activated brown adipose tissue in healthy men. $\mathrm{N}$ Engl J Med 360: 1500-1508, 2009.

9. Sell H, Deshaies Y and Richard D: The brown adipocyte: update on its metabolic role. Int J Biochem Cell Biol 36: 2098-2104, 2004.

10. Cannon B and Nedergaard J: Brown adipose tissue: function and physiological significance. Physiol Rev 84: 277-359, 2004.

11. Virtanen KA, Lidell ME, Orava J, et al: Functional brown adipose tissue in healthy adults. N Engl J Med 360: 1518-1525, 2009.

12. Zingaretti MC, Crosta F, Vitali A, et al: The presence of UCP1 demonstrates that metabolically active adipose tissue in the neck of adult humans truly represents brown adipose tissue. FASEB J 23: 3113-3120, 2009.

13. Cederberg A, Gronning LM, Ahren B, Tasken K, Carlsson P and Enerback S: FOXC2 is a winged helix gene that counteracts obesity, hypertriglyceridemia, and diet-induced insulin resistance. Cell 106: 563-573, 2001.

14. Puigserver P, Wu Z, Park CW, Graves R, Wright M and Spiegelman BM: A cold-inducible coactivator of nuclear receptors linked to adaptive thermogenesis. Cell 92: 829-839, 1998.

15. Seale P, Bjork B, Yang W, et al: PRDM16 controls a brown fat/skeletal muscle switch. Nature 454: 961-967, 2008.

16. Seale P, Kajimura S, Yang W, et al: Transcriptional control of brown fat determination by PRDM16. Cell Metab 6: 38-54, 2007.

17. de Jesus LA, Carvalho SD, Ribeiro MO, et al: The type 2 iodothyronine deiodinase is essential for adaptive thermogenesis in brown adipose tissue. J Clin Invest 108: 1379-1385, 2001.

18. Zhao J, Cannon B and Nedergaard J: Thermogenesis is beta3but not beta1-adrenergically mediated in rat brown fat cells, even after cold acclimation. Am J Physiol 275: R2002-R2011, 1998.

19. Tseng YH, Kokkotou E, Schulz TJ, et al: New role of bone morphogenetic protein 7 in brown adipogenesis and energy expenditure. Nature 454: 1000-1004, 2008.

20. Chomczynski P and Sacchi N: Single-step method of RNA isolation by acid guanidinium thiocyanate-phenol-chloroform extraction. Anal Biochem 162: 156-159, 1987.

21. Svensson PA, Gabrielsson BG, Jernås M, Gummesson A and Sjöholm K: Regulation of human aldoketoreductase 1C3 (AKR1C3) gene expression in the adipose tissue. Cell Mol Biol Lett 13: 599-613, 2008.

22. Manieri M, Murano I, Fianchini A, Brunelli A and Cinti S: Morphological and immunohistochemical features of brown adipocytes and preadipocytes in a case of human hibernoma. Nutr Metab Cardiovasc Dis 20: 567-574, 2009.

23. Sjöström L, Narbro K, Sjöström CD, et al: Effects of bariatric surgery on mortality in Swedish obese subjects. N Engl J Med 357: 741-752, 2007.

24. Harper JA, Dickinson K and Brand MD: Mitochondrial uncoupling as a target for drug development for the treatment of obesity. Obes Rev 2: 255-265, 2001.

25. Arch JR: The discovery of drugs for obesity, the metabolic effects of leptin and variable receptor pharmacology: perspectives from beta3-adrenoceptor agonists. Naunyn Schmiedebergs Arch Pharmacol 378: 225-240, 2008.

26. Seale P, Kajimura S and Spiegelman BM: Transcriptional control of brown adipocyte development and physiological function-of mice and men. Genes Dev 23: 788-797, 2009. 